\title{
The Feasibility of Gamma Irradiation for Developing Malaria Vaccine
}

\author{
M. Syaifudin“, D. Tetriana, Darlina and S. Nurhayati \\ Center for Technology of Radiation Safety and Metrology, National Nuclear Energy Agency \\ Jl. Lebakbulus Raya No. 49 Pasar Jumat, Jakarta, Indonesia
}

\section{ARTICLE INFO}

\section{Article history:}

Received 20 November 2010

Received in Revised form 11 July 2011

Accepted 01 August 2011

Keywords:

Antigenic protein

Gamma rays

Immune response

Malaria

Plasmodium sp

Sporozoite

\begin{abstract}
A B S T R A C T
Malaria, a plasmodial disease, causes more than one million deaths per year and has a significant public health impact. Improved access to prompt treatment with effective antimalarial drugs need to be conducted for prevention of infection in high risk groups. However, the parasite as causal agent has exhibited a potential danger of wide-spread resistances. This warning has directed attention to the study of alternative methods of protection against the disease, among them is to do the immunization. A deeper understanding of the nature and regulation of protective immune mechanisms against this parasite will facilitate the development of much needed vaccines. Developing a malaria vaccine remains an enormous scientific, technical, and financial challenge. Currently a vaccine is not fully available. Among the practical applications of radiobiological techniques that may be of considerable interest for public health is the use of ionizing radiation in the preparation of vaccines. Convincing data were reported that sporozoites of Plasmodium berghei irradiated with $\mathrm{X}$ - or gamma-rays, provide an antigenic stimulus effective to induce a protective immune response in mice and rats against subsequent sporozoite infection. Irradiated parasites are better immunogens than killed ones and although non-infective they are still metabolically active, as shown by continued protein and nucleic acid synthesis. There is a substantial number of data from human studies demonstrating that sporozoites attenuated by radiation are potent inducers of protective immunity and that they are safe and do not give rise to the asexual erythrocytic infections that cause malaria. This vaccine is relatively inexpensive to produce, easy to store, and transportable without refrigeration. A long-term effort and commitment to providing resources must be maintained and increased to achieve the goal of a malaria vaccine candidate where ionizing radiation as a tool to prepare is seemingly feasible.
\end{abstract}

(C) 2011 Atom Indonesia. All rights reserved

\section{INTRODUCTION}

Malaria is the most important disease of human in the world. As a whole, it is estimated that 500 million people are infected with the disease. This parasitic disease kills 2.7 million people across the world each year. In the past more than one-third of the total world populations were exposed to malaria infection and at least half the people who died from all causes were probably killed directly or indirectly by malaria [1]. This disease has a tremendous impact on human health and it is a major impediment for social and economic development of nations in malaria-endemic areas, particularly in sub-Saharan Africa and Asia. Malaria is also a major problem for young children and pregnant women [2]. The health implications of

\footnotetext{
* Corresponding author.

E-mail address: mukh_syaifudin@batan.go.id
}

malaria in tropical Africa are reflected in the exceptionally high mortality and morbidity rates in rural areas. In West Africa at least $10 \%$ of deaths below five years of age are due to malaria. While in unprotected rural communities the infant mortality rate from all causes, in which malaria plays a major role, may reach even $50 \%$. The highlands of tropical Africa are occasionally swept by severe malaria epidemics, which sometimes cause as high as $25 \%$ mortality [1].

Apart from tropical Africa, the disease is still endemic in large areas of the world including SouthEast Asia (SEA). In this region, ten out of 11 countries are malaria endemic. World Health Organization (WHO) estimates that of 40,213,000 Disability Adjusted Life Year (DALYs) in the world, 2,390,000 are in Asia (SEA 1,874,000 DALYs or $4.66 \%$ ) [2]. Although Asia contributes only about $6 \%$ of DALYs in the world, but malaria causes high morbidity and mortality in the endemic 
countries. The disease is deeply rooted in the poor communities, affecting national development and takes away major share of health budgets. The average laboratory confirmed cases were reported around 2.5 million annually where as probable malaria cases were around 20 million annually. An estimated of 1,326.5 million people, or $82.8 \%$ of the total population of SEA Region, are at risk of malaria. Out of which around $41.5 \%$ population at moderate to high risk of malaria, $41.7 \%$ are at low risk where as remaining $16.8 \%$ of population free from malaria. About $95 \%$ of the populations with moderate to high risk of malaria in SEA Region are living in Indonesia, Myanmar and Thailand [3].

According to the Department of Health that figuring the Indonesian Health Profile in 2004, malaria has spread almost uniform in all parts of the country mainly in the islands outside Java and Bali. It was estimated that $35 \%$ peoples or about 90 million populations were living in malaria risk areas [4]. However, the National Planning Agency claimed that the malaria incident was decreased since 2001, in part due to the implementation of Eradication Action on Malaria that started in April 2000. In Java and Bali the target number of malaria victims in 2002 was overed the target of indicator of Indonesia Health 2010, i.e. below 5 per 1000 peoples [5].

Improved access to prompt diagnosis and treatment of malaria needs to be combined with a multi-prolonged approach to prevention that should include vector control, insecticidetreated nets, and the use of drugs for prevention of infection in high risk groups (intermittent preventive treatment) [6,7]. However, the potential danger of wide-spread resistance of the vector to insecticides, and of drug resistance among plasmodia which infect humans is steadily increasing and has recently been stressed repeatedly. These warnings have again directed attention to the study of alternative methods of protection against the disease, among them to the immunization of population at risk. Furthermore, because of the limitations of diagnostic procedures based upon the detection of the contributing parasites in the blood, investigations have been intensified in recent years to develop immunobiological tests and to evaluate them in individual cases as well as in epidemiological surveys, and in determining the effects of control and treatment $[1,8]$.

\section{MALARIA AND VACCINE}

Malaria infection begins when a plasmodium infected female Anopheles mosquito bites a person and injecting these parasites, in the form of sporozoites, into the bloodstream. Once in the body, plasmodium has a complex life cycle. After is inoculated by mosquito biting, it will invade hepatocytes, transform into liver stages, and the subsequent liver stage development ultimately results in the release of pathogenic merozoites. These merozoites released from the liver cells in vesicles where eventually disintegrate, freeing the merozoites to enter the blood phase. In the bloodstream, the merozoites invade red blood cells (erythrocytes) and multiply again until the cells burst. Some of the infected blood cells leave the cycle of asexual multiplication. Instead of replicating, the merozoites in these cells develop into sexual forms of the parasite, called gametocytes, that circulate in the bloodstream. When a mosquito bites an infected human, it ingests the gametocytes, which develop further into mature sex cells called gametes. The cycle of human infection begins again when the mosquito bites another person (Fig. 1) $[9,10]$.

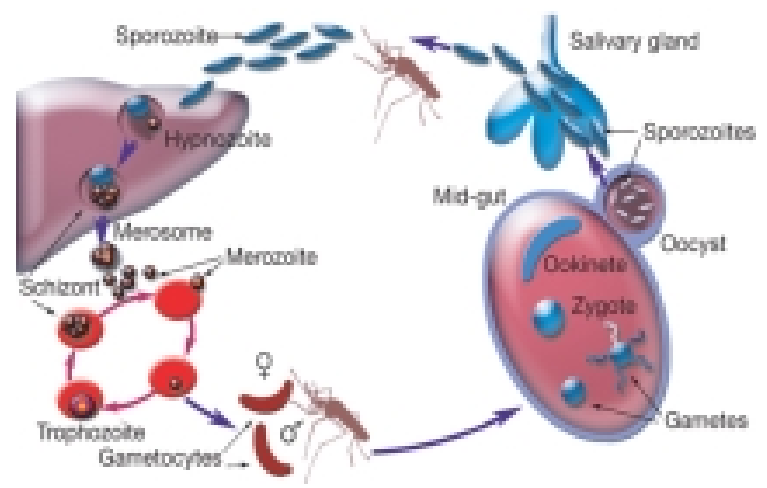

Fig. 1. Life cycle of Plasmodium sp. in the body of mosquito and infected host [9].

Vaccination is historically the most effective way to control infectious disease such as malaria. Vaccines are designed to stimulate the immune system to protect against micro-organisms such as viruses or bacteria, by introducing a small amount of the virus or bacteria into the body. When this foreign substance invades the body, the immune system activates certain cells to destroy the invader. If the body is re-invaded by the virus or bacteria in the future, the memory cells will be reactivated and respond faster and more powerfully to destroy the virus. It was suggested that active immunization may be of value in protection against some parasitic diseases, including malaria, and is mainly based on consideration of the natural history of the disease. 
A deeper understanding of the nature of protective immune mechanisms against malarial parasite will facilitate the development of much needed vaccines [10]. The first vaccination was performed by Edward Jenner who had noticed that dairy maids who had had cowpox infection did not succumb to the deadly smallpox infection that was claiming many lives at the time. The body's immune system is comprised of two arms that are antibody-mediated immunity and cell-mediated immunity. All vaccines developed in the last 50 years probably protect by stimulating a potent antibody response. However, for pathogens that live within cells of the body, where antibodies can't reach, it is likely that cell-mediated immunity is required for protection. Examples of such pathogens include HIV and malaria. Each of these is a huge global health problem claiming millions of lives each year for which there is no effective vaccine.

The development of a safe and efficacious malaria vaccine has remained an elusive goal for the past 80 years. However, recent years have witnessed a new impetus and the generation of conclusive evidence that effective immunity against Plasmodium falciparum infection, mild uncomplicated malaria, and severe disease can be induced through vaccination in children living in malaria-endemic areas. It is likely that the next decade will witness the first registration of a malaria vaccine. This vaccine is unlikely to provide complete protection, but it may become a useful tool to be added to the armory in the fight against malaria. Moreover, scientists exactly looking for in a malaria vaccine are that vaccine should require as few doses as possible to work and has to be cheap to make and administer. The vaccine should offer long-lasting, preferably life-long, protection against all parasite strains. Although it is the blood stage that causes clinical disease, an ideal vaccine would be a combination, able to work against multiple stages. As malaria is endemic in numerous developing countries, the vaccine would have to be stable in different temperatures and simple to administer. Ideally, vaccine use would use existing infrastructure used for infant immunization [11].

For about 25 years, scientists have tried to develop modern and recombinant 'subunit' malaria vaccines which is actually difficult. Most vaccines are based on attenuated or inactivated whole pathogens or material derived directly from the infectious agent. An effective vaccine against $P$. falciparum malaria remains one of the great challenges of medicine. Despite over one hundred years of effort, hundreds of millions of dollars in research, lifelong sacrifice from dedicated physicians and scientists and many promising experimental vaccines, there is no marketed vaccine to alleviate one of the great infectious scourges of humanity [12]. The past few years have witnessed the appearance of a significant increase of resources devoted to the development of malaria vaccines. Lessons have been learned on different approaches to the clinical development plan for a vaccine candidate, including different clinical trial phases and endpoints and methods to conduct them. Improved understanding of mechanisms underlying naturally acquired immunity, definitions of surrogate markers of protection, including improved in vitro assays and animal models, and strengthened capacity in malaria-endemic countries to conduct clinical trials would accelerate the development of malaria vaccines. The impact of this vaccine and subsequent products will need to be evaluated under program conditions in the context of comprehensive malaria control activities. Deployment of a safe and effective malaria vaccine administered in early childhood could become a major public health tool, particularly in sub-Saharan Africa [13].

Developing a malaria vaccine remains an enormous scientific, technical, financial, and political challenge. The plasmodium is a highly complex parasite, with a multi-stage life cycle, during which the parasite presents multiple antigens that show significant variability. The main hindrance to developing a vaccine is the choice of the antigen or antigens to be included. This process has often relied on the use of imperfect animal models, in vitro assays with significant limitations, and seroepidemiological assessments of naturally acquired immune responses in humans and their relationship to the risk of malaria infection or disease. Numerous associations between antigenspecific immune responses and the risk of infection or disease have been described. However, an association between a given immune response and the risk of malaria does not mean that those responses play a role in protection, as previous exposure to the parasite and development of responses to multiple antigens, most of which are unlikely to be linked to protection and are simply a marker of exposure, become an important confounding factor [13].

At present there are approximately 100 candidates of malaria vaccine in development, including pre-erythrocytic vaccines, blood-stage vaccines, anti-toxic vaccines, and transmissionblocking vaccines. Nevertheless, most of them are based on a small number of $P$. falciparum antigens $[14,15]$. Only a few candidates have entered clinical phase studies, and most are still in pre-clinical phases. The malaria community has understandably placed a major effort in the antigen selection process 
as the key to the development of a malaria vaccine. However, the results of the RTS,S candidate vaccine have highlighted the critical role that formulation and adjuvant technology may play in the successful development of a product. This vaccine, based on the circumsporozoite antigen, had failed to provide protection in numerous trials $[16,17]$, and following commonly held views, should have been abandoned a long time ago. However, formulation of that same antigen in a new adjuvant platform radically changed the outlook for this product, which has shown safety, immunogenicity, and induction of protection in challenge models with non-immune volunteers, hyper-immune adults, and semi-immune children living in malaria-endemic areas of Africa [16,18-20].

The overall results in human trials indicate a short duration protection. More recently RTS,S/AS02A vaccine has performed better in infants less than 1 year of age. Another example is a DNA vaccine using TRAP (thrombospondin-related adhesive protein) antigen with a string of T-cell epitopes using a prime-boost sequence with MVA (modified vaccinia virus Ankara) or FP9 which is attenuated pox virus followed by MVA as boosters. This induced high T-cell response and a substantial delay to parasitemia in sporozoite challenge studies. None of these results match the complete protection obtained with irradiated sporozoites in experimental animals and human volunteers [14]. Many researchers are developing a non-replicating, metabolically active, radiation-attenuated $P$. falciparum sporozoite vaccine. When such sporozoites are injected intravenously into mice, they enter the liver, but do not develop further. Interestingly, when 25,000-50,000 of these knockout sporozoites are injected into mice using different prime-boost protocols, they protect the animals when infected with wild type sporozoites. This protection is seen even two months after vaccination. The vaccine is stage-specific, since blood stage parasites when injected develop normally. Protection is also seen when sporozoites are introduced through mosquito bites. Thus, proof of principle for a genetically altered whole-parasite based vaccine has been established [21,22].

One main obstacle in malaria vaccine discovery is the absence of sterilizing immunity during natural infection. The corresponding wholeorganism vaccines mimic an acute pathogen infection, which were known to function as a natural vaccination after the host immune system resolved the first infection. There is no such model of acquired immunity against the plasmodium parasite. Yet, a malaria vaccine will only become an efficient public health tool if it provides protection for several years with no more than three immunizations. One potential, yet challenging, solution to this problem may be the composition of vaccine strategies that aim at inducing protective immune responses against immunological silent plasmodium life cycle stages, i.e. those that are not the typical targets of naturally acquired immunity. Recent insights into the parasite biology and technological advancements open the possibility to explore such alternative vaccine strategies.

Vaccine strategies against malaria is that natural transmission to the human host may be reduced by high titers of sporozoite-neutralizing antibodies that act prior to hepatocyte entry. In addition, vaccination with sporozoite antigens may induce cell-mediated responses to the infected hepatocyte. The progression of pathogenic blood stages can be reduced during the brief phase of merozoite entry into erythrocytes. P. falciparuminfected erythrocytes adhere to endothelial cells in capillaries and the placenta through parasiteencoded surface proteins that eventually lead to antibody recognition. Maturation of liver-stage schizonts and ookinete penetration of the mosquito midgut represent two immunology silent stages of the plasmodium life cycle. Genetically attenuated parasites elicit long-lasting complete protection in experimental models. These genetically defined parasites are inoculated as sporozoites and invade and transform normally, but arrest during subsequent liver-stage development. At this time they likely display protective antigens in the context of major histocompatibility complex (MHC) class I presentation that, in turn, activate interferon-csecreting effector $\mathrm{T}$ cells. Two partially redundant ookinete surface proteins, Pfs25 and Pfs28, constitute attractive targets for the development of transmission blocking vaccines. Targets for vaccine development include surface proteins or adhesion proteins of invasive stages [22].

\section{DEVELOPING VACCINE WITH NUCLEAR TECHNOLOGY}

Among the practical applications of radiobiological techniques that may be of considerable interest for public health is the use of ionizing radiation in the preparation of vaccines. Attempts of vaccine production by exposing various kinds of micro-organisms including viruses, bacteria and fungi, as well as unicellular and multicellular parasites to ionizing radiation have been reported in scientific periodicals from time to time. Radiation attenuated vaccines have been demonstrated to be an effective means of 
controlling certain parasitic infections such as malaria. Although many parasitic diseases are known to produce a high degree of acquired immunity, it has been impossible in the past to reproduce this immunity using artificially prepared vaccines, the striking exception being the radiation attenuated vaccines. The successful use of irradiated plasmodium as a vaccine depends on finding a radiation dose which will significantly reduce the pathogenic effect of the larvae without seriously impairing their immunogenic power [23].

Gamma radiation is widely used by many researchers to inactivate parasite for the preparation of vaccines, instead of traditional heat or chemical methods of inactivation, appears to create a vaccine that is more effective than so-called "killed" vaccines against disease, and has the added advantage of a longer storage life than "live" vaccines. Irradiation is a technically simple process that retains structural features of the microbial pathogen without destroying the natural antigens or the intrinsic adjuvants. Therefore, a strong immune response is induced in the vaccinated host. Irradiation destroys the DNA, making the microorganism unable to replicate so it cannot establish an infection, but some residual metabolic activity may survive, so the irradiated microorganism can still find its natural target in the host. Vaccination with a freeze-dried powder formulation of the irradiated bacteria, a product with the potential to be easily and inexpensively stored and transported then reconstituted just before use, was also shown to protect mice against lethal infection [23]. In experiments with mice, the researchers in Infectious Diseases Section, VA San Diego Healthcare System USA demonstrated that a vaccine made with irradiated Listeria monocytogenes bacteria provided much better protection against disease than vaccine made from heat-killed bacteria. Irradiation is a technically simple process that retains structural features of the bacterial pathogen without destroying the natural antigens or the intrinsic adjuvants. Therefore, a strong immune response is induced in the vaccinated host.

Since 1966, the International Atomic Energy Agency (IAEA), in collaboration with the Food and Agricultural Organization of the United Nations, has had a co-ordinated research programme on the application of nuclear techniques to human parasitology. It soon became obvious that the similarity of certain parasitic infections in man and animals as well as the existence of cross-infections would make it extremely difficult to keep any strict and unnatural species barriers. The apparent similarity, for instance, between human and animal trematodes like Trypanosoma and plasmodium clearly suggests the extension of these studies to human parasitic diseases. The recommendations of the meeting between IAEA and WHO several years ago urged these two international organizations that a co-ordinated collaborative research programme be initiated on the use of nuclear techniques in preparation of vaccines against human parasitic diseases, with the highest priority being given to malaria. There are essentially two distinct ways in which nuclear techniques can be employed in research performed under this co-ordinated programme : the use of ionizing radiation for attenuation of the parasitic organisms; and the use of radioisotopes as tracers in studies on the physiology of the parasites and on the host-parasite relationships.

Subjects recommended for research using a more empirical approach are as follow : further immunization trials with radiation attenuated vaccines on various host-parasite systems with both homologous and heterologous combinations of immunizing an challenging antigenic variants; determination of optimum dosage of irradiated parasites; number of boosters; spacing of vaccination; characteristic, duration and species specificity of the protection; occurence of undesired side effects following vaccination; radiosensitivity of the parasites at their different stages of development; and optimum range of radiation doses to be applied for attenuation.

Furthermore recommendations for basic studies those are to contribute to the general understanding of the host-parasite interactions in human parasitic infections and the physiology of the parasites include subjects such as: methods for labelling of parasites with radioactive isotopes and for determining the nature and location of their antigens; morphological and physiological alterations in parasites induced by irradiation; survival and localisation of irradiated parasites in the inoculated host; existence of natural antibodies against parasites; the relative role of cell mediated versus antibody mediated immunity in host defense mechanism; passive transfer of immunity into sublethally irradiated recipient animals; induction and/or selection of genetic mutant parasites that are less pathogenic and thus more suitable for vaccination; and metabolism of parasites and the essential requirements for their cultivation in vitro.

In 1967, Nussenzweig reported that immunizing mice with radiation-attenuated Plasmodium berghei sporozoites protected them against challenge with fully infectious sporozoites [24]. This rodent study provided the impetus for human studies. During the 1970s, Clyde DF and 
colleagues established that immunizing human volunteers with bites of irradiated mosquitoes carrying $P$. falciparum sporozoites in their salivary glands could protect volunteers against challenge with fully infectious $P$. falciparum sporozoites [25]. These studies demonstrated that a malaria vaccine offering sterile protective immunity was possible. However, the only way to produce sporozoites at that time was to infect a volunteer with $P$. falciparum, treat the volunteer with doses of chloroquine to suppress but not eliminate the parasite, allow gametocytes to develop and then feed mosquitoes on the volunteer.

Even if one could produce sporozoites in adequate numbers by this method, it was considered clinically, technically and logistically impractical to immunize large numbers of individuals with an irradiated sporozoite vaccine, in large part because the sporozoites had to be delivered alive, either by the bite of infected mosquitoes or by an intravenous injection, as was done with mice. Potential solutions to these problems, although not necessarily recognized at the time as being related to developing an attenuated sporozoite vaccine, were being reported. In 1975, a method for culturing $P$. falciparum in vitro was reported [26,27], followed by a method for producing gametocytes from these cultures in 1986 [28]. In 1986, it was reported that humans could be infected by the sporozoites produced in mosquitoes that had fed on these in vitro cultures [29]. There was therefore a way to produce sporozoites without the difficulties of in vivo production of gametocytes in humans.

Convincing data were reported during the past few years that relatively small numbers of sprozoites of $P$. berghei irradiated with $\mathrm{X}$ - or gamma-rays, provide an antigenic stimulus effective to induce a protective immune response in both mice and rats against subsequent sporozoite infection, but not against infection with blood forms of the parasite. For protection of the animals against an infecting dose of blood forms, a vaccination is necessary with parasitized red blood cells that have previously been exposed to ionizing radiation. According to preliminary studies carried out in monkeys, the results on rodent parasites cannot be extrapolated to primate plasmodia, though, in one of the experiments a definite protection has been obtained with four intravenous inocula of infected and irradiated red blood cells. Further investigations with $P$. knowlesi have suggested that one of the reasons why irradiated parasites are better immunogens than killed ones is that, although noninfective, they are still metabolically active, as shown by continued protein and nucleic acid synthesis. It has become evident that normal multiplication and infectivity of trypanosomes can be suppressed by a small fraction of the dose required to kill the parasites. Thus irradiation may enable one to take advantage of the special immunological properties of living parasites. At the same time, the pathogenic effects of a vaccine prepared from parasites with an unimpaired reproductive capacity would be obviated. In spite of this promising hypothesis, the research on the possible employment of radiation attenuation for the control of trypanosomiasis has been resumed only recently [30].

Large-scale production of attenuated parasite vaccine may indeed become feasible, because some challenges, such as sterility, cryopreservation, and route of immunization, have either already been met or are under active investigation [12]. Other roadblocks related to the safety and batch-to-batch variation of genetically undefined irradiated sporozoites have recently been removed in the rodent malaria model system by the generation of genetically attenuated parasites (GAPs) [21]. Although translation to the $P$. falciparum system may take several years, early human challenge studies with irradiated sporozoites indicate that complete attenuation of liver-stage development elicits protection to date the gold-standard in $P$. falciparum vaccine development. GAPs differ from gamma-irradiated sporozoites in their consistent production, genetic stability, and higher potency [31,32]. A fundamental issue is whether natural exposure to plasmodium transmission would boost GAP-induced immune responses. If this was the case a GAP vaccine would be feasible for individuals from malaria-endemic countries. Otherwise only short-term visitors would benefit and GAPs would fall into the category of 'boutique vaccines'.

A critical focus of vaccine development process has been to determine the minimum dose of radiation that adequately attenuates all sporozoites, and thus ensures that the vaccine will not cause malaria. Researchers are also interested in determining the highest dose of radiation that leaves the sporozoites able to elicit optimal protective immunity. For this, Chattopadhyay R. et al. (2008) have studied in the Plasmodium yoelii rodent model system [33]. Exposure to $100 \mathrm{~Gy}$ completely attenuated $P$. yoelii sporozoites. They also demonstrated that immunization of mice intravenously with 3 doses of $750 P$. yoelii sporozoite that had received $200 \mathrm{~Gy}$, double the radiation dose required for attenuation, resulted in $100 \%$ protection. These results support the contention that a radiation attenuated sporozoite 
vaccine for malaria will be safe and effective at a range of radiation doses.

Presently there is no malaria vaccine available to travellers or individuals living in malaria endemic areas. A recent promising vaccine to undergo clinical trials in Africa is the $P$. falciparum circumsporozoite (CS) protein construct with recombinant hepatitis particles (RTS,S). Previously, this vaccine induced $50-60 \%$ pre-erythrocytic protection against homologous $P$. falciparum challenge in naïve volunteers [16,34]. However, in Gambian adults, this vaccine did not induce similar patterns of immunity and protection was short-lived $[18,19]$. It could be argued that this vaccine, targeting the highly polymorphic CS antigen, worked as expected, since a heterogenous population of parasites exist in endemic areas. McConkey and colleagues recently reported highly encouraging results on the induction of substantial $P$. falciparum specific CD4 and CD8 T cell levels in naive humans using Prime-boost [35]. Such protocols, if combined with high selectivity in antigen and variant/conserved epitope inclusion, may help overcome the problems of variant-specific immune evasion. Indeed, the most potent vaccine for malaria endemic areas may prove to be a multiantigen, multi-stage combination.

However, identification of new and conserved antigens alone may not be sufficient for a vaccine that is successful in malaria endemic areas, as acute malaria infection is associated with $\mathrm{T}$ cell immunosuppression with a variety of generalized immunological changes, some of which are discussed above. An additional problem common to all vaccines is that in the field, they may preferentially re-stimulate pre-existing immunity induced by past infection with a bias towards a specific, and not necessarily protective, cytokine secretion pattern. It is hoped that as new vaccine approache and understanding of the mechanisms of immune evasion at the host parasite interphase progress, elements of its design will provide an effective vaccine for use in malaria endemic populations [35].

\section{DOSE OF IRRADIATION AS CRUCIAL FACTOR}

The dose of radiation used to attenuate plasmodium is the most crucial factor. Therefore, to determine the dose of gamma-irradiation which will produce attenuated parasites without affecting the pre-erythrocytic and erythrocyte stabilities is the ultimate goal of many studies on irradiation vaccine. It is thought that the amount of radiation required to render the parasites nonviable is about $150 \mathrm{~Gy}$ for an inoculum of certain number of parasites [12]. There is also evidence that exo-erythrocytic stages may be more susceptible to gamma-rays than are blood parasites. It means that a most important step is to determine minimum dose of irradiation required to adequately attenuate each sporozoite.

Scientists in many parts of the world have created harmless versions of malaria-causing parasite by applying radiation technology, and are using them to create a new malaria vaccine that could be more effective than the ones already in existence. Researchers at the National Institute of Standards and Technology (NIST) in USA used their expertise to help Maryland-based biotech firm Sanaria Inc., which is creating the new vaccine, create these parasites. The researchers already have extensive knowledge relating to measuring the right doses of radiation for other procedures. The scientists from the NIST are going to continue to work with Sanaria Inc. and perform quality assurance tests to make sure that the dose is being given to the mosquitoes correctly [1].

Decades ago, scientists had discovered that volunteers built up high levels of protection to malaria after being exposed to mosquitoes containing live, radiation-weakened parasites. Since then they had been striving to use vaccines based on whole living parasites. However, the developing technology for extracting weakened parasites from their mosquito carriers has introduced recently. In the manufacturing process, live mosquitoes containing the parasite are exposed to gamma rays. To ensure that the parasites are sufficiently weakened for the vaccine, yet remain alive, they must be exposed to a radiation dose of at least $150 \mathrm{~Gy}$, but not much more. The researchers have revealed that to be safe for human trials, all mosquitoes must get their minimum radiation dose of 150 Gy. NIST researchers will remain associated with the manufacturing of the vaccine by doing regularly scheduled quality-assurance tests to ensure the desired dose is being delivered to the mosquitoes. It is only at this dose that they can be sure that the parasites are weak enough for the vaccine, but are still alive [36].

Experiments conducted by Ferreira-da-Cruz et al. [37] in Brazil provide evidences that a $200 \mathrm{~Gy}$ gamma-irradiation is able to abolish the original replication of erythrocytic forms of the Palo Alto $P$. falciparum strain, probably by inactivating their infectivity. According to their data, 100 or $150 \mathrm{~Gy}$ irradiation doses were enable to inactivate the 
parasite, despite the reduction of parasitemia, suggesting the existence of heterogenous plasmodial populations as concerns susceptibility to irradiation and pointing to the possibility that the same dose could inactivate parasites from other P. falciparum strains. Beside that, in view of the nonexistence of defense immune mechanisms in the in vitro culture, it is reasonable to suppose that the dose required to abolish infectivity in these conditions is higher than that expected to inactivate the parasite in vivo, fact that increases the safety of the proposed dose of irradiation. Nevertheless, studies of the effects of gamma-irradiation on the in vivo infectivity of $P$. falciparum in non human primates are necessary to clarify this question. If proven to be safe, this methodology could rescue blood bags eventually contaminated with $P$. falciparum, with low costs and no risk of inducing transfusional malaria, and help solving the problem of the chronic deficit of blood in malaria endemic areas.

Research on malaria in BATAN is being focused on the development of irradiation vaccine that was started in 2005 and included in Main Focus through Research Project entitled "The Controlling Infectious Diseases with Nuclear Technology" [38]. In preliminary step of research the determination of optimal gamma rays dose for attenuating parasite to get basic material of vaccine was done by using $P$. berghei as model. In erythrocytic stage, the gamma attenuated parasite was effective in eliciting immune response and supressing its infectious pattern as approved by longer prepatent periods, the lower percentage of parasitemia and less mortality of mouse compared to those of non irradiated plasmodium. Irradiation dose of 75-125 Gy were not enable to attenuate plasmodium where the increasing of parasitemia was seen up to $16-22$ days post innoculation of irradiated plasmodium.

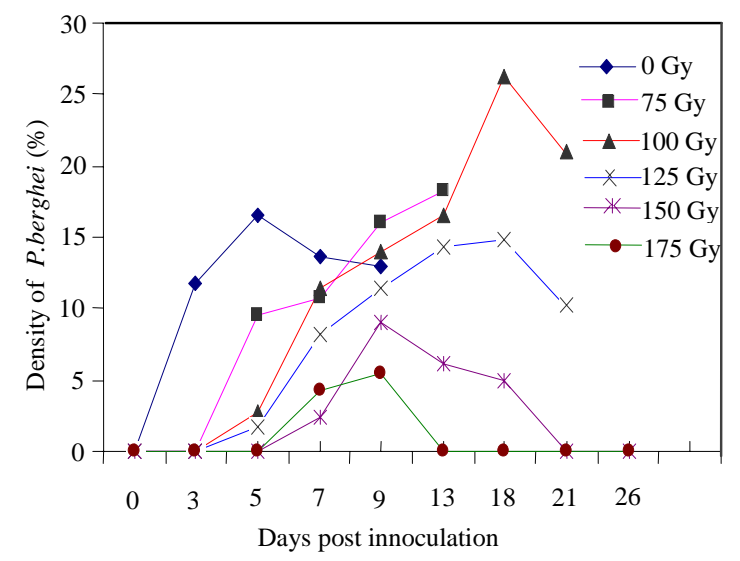

Fig. 2. Parasitemia in blood of Swiss mouse post inoculation of attenuated $P$. berghei at several doses of gamma rays [39].
Whereas irradiation dose of 150-175 Gy were enable to suppress the infected erythrocyte cells and supported by the fact that long pre-patent, low parasitemia and lethality of mouse were found (Fig. 2) [39]. The second booster at two weeks after the first innoculation of 150 Gy irradiated plasmodium could elicit immune response and resulted in low density of plasmodium in the blood.

The most effective malaria vaccine so far has been the irradiated sporozoite model. This vaccine, as it is now made, requires $P$. falciparum infected mosquitoes to be exposed to non-lethal levels of gamma irradiation sufficient to impair the development of the sporozoites in the salivary glands. This latter is currently conducted in BATAN in order to create irradiation sporozoite vaccine for malaria [38].

\section{CONCLUSION}

Malaria remains one of the most devastating infectious diseases that threaten humankind. Many scientists believe that the development and implementation of effective malaria vaccines, especially against the predominant species $P$. falciparum and $P$. vivax, will be critical to achieve malaria eradication. With malaria vaccines potentially within reach, it is important that the international community continue to support and increase investments in malaria vaccine research [12].

The first, and as yet unsurpassed, success in inducing protective immune responses against malaria was achieved with irradiated sporozoites in a rodent malaria model system Nussenzweig [24]. Immunization of mice with three doses of gammairradiated sporozoites results in attenuated liver-stage development and elicits complete sustained protection against sporozoite challenge. Analogous to other live-attenuated vaccines, arrested plasmodium liver stages likely induce protective cell-mediated immune responses against the entire antigenic repertoire of the liver stage and may be the most potent malaria vaccine. Exposure of humans to radiation-attenuated $P$. falciparum sporozoites produced at multiple different sites was proven to elicit a high level of reproducible protective immunity in human volunteers at multiple locations. Much remains to be accomplished, but the path forward is clear. They are encouraged by the support of so many recognized experts in a wide spectrum of disciplines who have offered constructive advice and assistance to this nascent endeavor [23]. 
Many factors make malaria vaccine development are challenging. The size and genetic complexity of the parasite mean that each infection presents thousands of antigens to the human immune system. The parasite also changes through several life stages even while in the human host, presenting different antigens (proteins) at different stages of its life cycle. For many infectious diseases it is possible to produce an attenuated (harmless) version of the pathogen or a pathogen subunit that will lead to protective immunity without causing disease. Although this is technically possible such as irradiated malaria sporozoites given by infected mosquito bite can lead to protective immunity, it is impractical to do this on a large scale [16].

In fact, although malaria vaccine development to date has met with only limited success, there are several lines of evidence that suggest that a malaria vaccine is possible: first, current clinical studies have shown that new candidate vaccines can induce complete protection against malaria infection; second, complete protection against malaria can be induced by infecting volunteers with irradiated malaria parasites; third, people living in endemic areas who have been multiply exposed to malaria develop immunity against severe malaria disease; and fourth, antibodies purified from life-long residents of endemic areas can be transferred into other individuals and can confer some protection against the effects of malaria infection [40].

The genetic heterogeneity is one of the main causes of the difficulties in developing an effective malaria vaccine. Segregation analysis identified that the nature of genetic factors are involved in the expression of human diseases. Moreover, this genetic variant closely associated with almost 30 percent reduced risk of developing severe malaria. There is an increasing evidence of genetic predisposition to both susceptibility and outcome in this infectious disease. Emerging research also demonstrates that diet, pollution, and other environmental triggers can alter both the function and expression of human genes and lead to a heightened disease risk of malaria. Robust epidemiological evidence showed that host genetic factors are important determinants of the outcome of interactions between host and pathogen. Genetic variation in the parasite is central to the pathogenesis of the organism, as allelic variability in different clones is thought to facilitate immune evasion. This in turn has significantly impeded progress towards the development of malaria vaccine [41]. Based on these facts, the development of malaria vaccine should be conducted regionally, not globally. Therefore it seems that we should develop our own malaria vaccine that is specific for Indonesian or at least SEA populations.

\section{REFERENCES}

1. L. Sztanyik, Radio-vaccines to combat human parasitic diseases, Division of Life Sciences, USA. http://www.iaea.org/publication/magazines/Bulletin/Bull153/1530558-2032.pdf.

2. D. Syafruddin, J.E. Siregar and S. Marzuki, Mol. Biochem. Parasitol. 104 (1999) 185.

3. World Health Organization/Roll Back Malaria, Malaria early warning systems - concepts, indicators and partners. A framework for field research in Africa, Geneva (2001) 47.

4. Badan Perencanaan Pembangunan Nasional (Bappenas), Laporan Perkembangan Pencapaian Tujuan Pembangunan Millennium Development Goals, Jakarta (2004). http://www.bappenas.go.id/index.php?module $=$ ContentExpress $\&$ func $=$ display $\&$ ceid $=853$.

5. Departemen Kesehatan Republik Indonesia, Profil Kesehatan 2002, Jakarta (2004).

6. S. Gunawan, Epidemiologi Malaria, in: Harijanto PN (Editor): Malaria Epidemiologi, Patogenesis, Manifestasi Klinis, \& Penanganan, Penerbit Buku Kedokteran EGC, Jakarta (2000) 1.

7. S.I. Hay, C.A. Guuerra, A.J. Tatem, A.M. Noor and R.W. Snow, Lancet. Infect. Dis. 4 (2004) 327.

8. J.G. Breman, American J. Tropical Medicine and Hygiene 64 (2001) 1.

9. E.A. Winzeler, Nature 455 (2008) 751.

10. K. Baer, C. Klotz, S.H. Kappe, T. Schnieder and U. Frevert, PLoS Pathogens 3 (2007) e171.

11. B. Mons, Annals of Tropical Medicine and Parasitology 91 Suppl. 1 (1997) 55.

12. T.C. Luke and S.L. Hoffman, The J. Exp. Biol. 206 (2003) 3803.

13. C. Guinovart and P.L. Alonso, American J. Tropical Medicine and Hygiene 77 Suppl. 6 (2007) 276.

14. World Health Organization, Candidate Malaria Vaccines in Pre-Clinical Development, Geneva (2006). 
15. Z.H. Reed, M. Friede and M.P. Kieny, Curr. Mol. Med. 6 (2006) 231.

16. J.A. Stoute, M. Slaoui, D.G. Heppner, P. Momin, K.E. Kester, P. Desmons, B.T. Wellde, N. Garcon, U. Krzych and M. Marchand, N. Engl. J. Med. 336 (1997) 86.

17. D.M. Gordon, T.W. McGovern, U. Krzych, J.C. Cohen, I. Schneider, R. LaChance, D.G. Heppner, G. Yuan, M. Hollingdale and M. Slaoui, J. Infectious Diseases 171 (1995) 1576.

18. K.E. Kester, D.A. McKinney, N. Tornieporth, C.F. Ockenhouse, D.G. Heppner, T. Hall, U. Krzych, M. Delchambre, G. Voss, M.G. Dowler, J. Palensky, J. Wittes, J. Cohen and W.R. Ballou, J. Infectious Diseases $\mathbf{1 8 3}$ (2001) 640.

19. K.A. Bojang, P.J. Milligan, M. Pinder, L. Vigneron, A. Alloueche, K.E. Kester, W.R. Ballou, D.J. Conway, W.H. Reece, P. Gothard, L. Yamuah, M. Delchambre, G. Voss, B.M. Greenwood, A. Hill, K.P. McAdam, N. Tornieporth, J.D. Cohen and T. Doherty, Lancet 358 (2001) 1927.

20. P.L. Alonso, J. Sacarlal, J.J. Aponte, A. Leach, E. Macete, J. Milman, I. Mandomando, B. Spiessens, C. Guinovart, M. Espasa, Q. Bassat, P. Aide, O. Ofori-Anyinam, M.M. Navia, S. Corachan, M. Ceuppens, M.C. Dubois, M.A. Demoitie, F. Dubovsky, C. Menendez, N. Tornieporth, W.R. Ballou, R. Thompson and J. Cohen, Lancet 364 (2004) 1411.

21. A.K. Mueller, M. Labaied, S.I. Kappe and K. Mutuschewski, Nature 433 (2005) 164.

22. P.L. Alonso, J. Sacarlal, J.J. Aponte, A. Leach, E. Macete, P. Aide, B. Sigauque, J. Milman, I. Mandomando, Q. Bassat, C. Guinovart, M. Espasa,. S. Corachan, M. Lievens, M.M. Navia, M.C. Dubois, C. Menendez, F. Dubovsky, J. Cohen, R. Thompson and W.R. Ballou, Lancet 366 (2005) 2012.

23. Anonymous, Using gamma radiation preserves T-cell responses in bacterial vaccine, Medical Research News. http://health.ucsd.edu/news/ 2006/07_25_Raz.htm. Retrieved in July (2006)

24. R. Nussenzweig, J. Vanderberg, H. Most and C. Orton, Nature 216 (1967) 160.

25. D.F. Clyde, V.C. McCarthy, R.M. Miller and W. Woodward, American J. Tropical Medicine and Hygiene 24 (1975) 397.
26. W. Trager, Living Together, in: The Biology of Animal Parasitism, Plenum Press, New York (1986).

27. D.J. Haynes, C.L. Diggs, F.A. Hines and R.E. Desjardins, Nature 263 (1976) 767.

28. C.C. Campbell, W.E. Collins, P. NguyenDinh, A. Barber and J.R. Broderson, Science 217 (1982) 1048.

29. J.D. Chulay, I. Schneider, T.M. Cosgriff, S.L. Hoffman, W.R. Ballou, I.A. Ouakyi, R. Carter, J.H. Trosper and W.T. Hockmeyer, American J. Tropical Medicine and Hygiene 35 (1986) 66.

30. K. Matuschewski and A.K. Mueller, FEBS J. 274 (2007) 4680.

31. S.L. Hoffman, L.M. Goh, T.C. Luke, I. Schneider, T.P. Le, D.L. Doolan, J. Sacci, P. De La Vega, M. Dowler and C. Paul, J. Infectious Diseases 185 (2002) 1155.

32. O. Jobe, J. Lumsden, A.K. Mueller, J. Williams, H. Silva-Rivera, S.H.I. Kappe, R.J. Schwenk, K. Matuschewski and U. Krzych, J. Infectious Diseases 196 (2007) 599.

33. R. Chattopadhay, S. Conteh, M.L. Li, E.R. James, J.E. Epstein and S.L. Hoffman, Vaccine 27 (2009) 3675.

34. J.A. Stoute, K.E. Kester, U. Krzych, B.T. Wellde, T. Hall, K. White, G. Glenn, C.F. Ockenhouse, N. Garcon, R. Schwenk, D.E. Lamar, P. Sun, P. Momin, R.A. Wrrtz, C. Golenda, M. Slaoui, G. Wortmann, C. Holland, M. Dowler, J. Cohen and W.R. Ballou, J. Infectious Diseases 178 (1998) 1139.

35. S.J. McConkey, W.H. Reece, V.S. Moorthy, D. Webster, S. Dunachie, G. Butcher, J.M. Vuola, T.J. Blanchard, P. Gothard, K. Watkins, C.M. Hannan, S. Everaere, K. Brown, K.E. Kester, J. Cummings, J. Williams, D.G. Heppner, A. Pathan, K. Flanagan, N. Arulanantham, M.T.M. Roberts, M. Roy, G.L. Smith, J. Schneider, T. Peto, R.E. Sinden, S.C. Gilbert and A.V.S. Hill, Nature Medicine 9 (2003) 729.

36. Anonymous, Radiation Weakened Parasites: Possible New Malaria Vaccine? http://www.medindia.net/news/RadiationWeakened-Parasites-Possible-New-MalariaVaccine-29169-1.htm. Retrieved in November (2007). 
37. M.F. Ferreira-da-Cruz, A. Teva, E.M. Erikada-Cruz, L.G. Santos and D.R.C. Tadeu, Memorias do Instituto Oswaldo Cruz 92 (1997) 137.

38. M. Syaifudin, R. Marialina, M.E. Aritonang and D. Tetriana, Identification of $M$. tuberculosis with duplex PCR and analysis of its resistance to drugs with radioactive SSCP, Proceeding of Scientific Presentation on Radiation and Environmental Safety XI, Jakarta, December 21 (2005).

39. Darlina and D. Tetriana, Infectious power of erithrocytic stage of Plasmodium berghei irradiated with gamma rays, Proceeding of Scientific Presentation on Radiation and Environmental Safety XIII, Jakarta, December 21 (2007).

40. D.S. Pouniotis, O. Proudfoot, G. Minigo, J.L. Hanley and M. Plebanski, J. Postgraduate Med. 50 (2004) 30.

41. B.A. Biggs, L. Gooze, K. Wycherley, W. Wollish, B. Southwell, J.H. Leech and G.B. Brown, Proceeding of the National Academy of Sciences USA 88 (1991) 9171. 